Technical Note

\title{
Sparse SAR Imaging and Quantitative Evaluation Based on Nonconvex and TV Regularization
}

\author{
Zhongqiu Xu ${ }^{1,2, * \mathbb{D}}$, Bingchen Zhang ${ }^{1}$, Guoru Zhou ${ }^{1,2}$, Lihua Zhong ${ }^{1}$ and Yirong $\mathrm{Wu}^{1}$ \\ 1 Key Laboratory of Spatial Information Processing and Application System Technology, \\ Chinese Academy of Sciences, Beijing 100190, China; zhangbc@aircas.ac.cn (B.Z.); \\ zhouguoru20@mails.ucas.ac.cn (G.Z.); lhzhong@mail.ie.ac.cn (L.Z.); wyr@mail.ie.ac.cn (Y.W.) \\ 2 University of Chinese Academy of Sciences, Beijing 100190, China \\ * Correspondence: xuzhongqiu18@mails.ucas.ac.cn; Tel.: +86-182-1050-7696
}

Citation: Xu, Z.; Zhang, B.; Zhou, G.; Zhong, L.; Wu, Y. Sparse SAR

Imaging and Quantitative Evaluation Based on Nonconvex and TV Regularization. Remote Sens. 2021, 13, 1643. https://doi.org/10.3390/ rs13091643

Academic Editor: João Catalão Fernandes

Received: 22 March 2021

Accepted: 20 April 2021

Published: 22 April 2021

Publisher's Note: MDPI stays neutral with regard to jurisdictional claims in published maps and institutional affiliations.

Copyright: (c) 2021 by the authors. Licensee MDPI, Basel, Switzerland. This article is an open access article distributed under the terms and conditions of the Creative Commons Attribution (CC BY) license (https:// creativecommons.org/licenses/by/ $4.0 /)$.

\begin{abstract}
Sparse signal processing has been used in synthetic aperture radar (SAR) imaging due to the maturity of compressed sensing theory. As a typical sparse reconstruction method, $L_{1}$ regularization generally causes bias effects as well as ignoring region-based features. Our team has proposed to linearly combine the nonconvex penalty and the total variation (TV)-norm penalty as a compound regularizer in the imaging model, called nonconvex and TV regularization, which can not only reduce the bias caused by $L_{1}$ regularization but also enhance point-based and region-based features. In this paper, we use the variable splitting scheme and modify the alternating direction method of multipliers (ADMM), generating a novel algorithm to solve the above optimization problem. Moreover, we analyze the radiometric properties of sparse-signal-processing-based SAR imaging results and introduce three indexes suitable for sparse SAR imaging for quantitative evaluation. In experiments, we process the Gaofen-3 (GF-3) data utilizing the proposed method, and quantitatively evaluate the reconstructed SAR image quality. Experimental results and image quality analysis verify the effectiveness of the proposed method in improving the reconstruction accuracy and the radiometric resolution without sacrificing the spatial resolution.
\end{abstract}

Keywords: sparse SAR imaging; nonconvex and TV regularization; image quality assessment; radiometric property; spatial resolution

\section{Introduction}

SAR is the major modern microwave imaging technology in remote sensing and is capable of producing high-resolution images of the Earth's surface. Compared with optical sensing technology, SAR is an active sensing technology and has all-time and all-weather observing ability. In recent years, with the development of compressed sensing, sparse signal processing has been widely used in SAR imaging, forming a novel strategy of SAR imaging named sparse SAR imaging. The sparse SAR imaging system can reconstruct a sparse signal with far fewer samples than that required by Nyquist-Shannon theory; in addition, it can also improve the reconstruction performance under full sampling conditions [1-3]. Researchers have applied sparse SAR imaging to a variety of SAR imaging modes, including stripmap SAR, ScanSAR, spotlight SAR and TOPS SAR modes $[2,4,5]$.

$L_{1}$ regularization is a common reconstruction model in compressed sensing theory. The $L_{1}$ regularization-based SAR imaging model was first proposed in [6]. Then the 1dimension (1-D) and 2-dimension (2-D) $L_{1}$ regularization-based SAR imaging models were proposed in $[7,8]$. When implemented in sparse SAR imaging, $L_{1}$ regularization can suppress the sidelobes as well as improving the target background ratio. However, there exist two problems in $L_{1}$ regularization. Firstly, the $L_{1}$-norm penalty is convex, and the analytic solution of the sparse signal reconstruction algorithm based on $L_{1}$ regularization is a biased estimation according to the related theory in statistical signal processing $[9,10]$, which leads to the underestimation of targets' reflectivity, affecting the reconstruction 
accuracy. Secondly, $L_{1}$ regularization can only enhance point-based features in SAR images while ignoring region-based features, which means its reconstruction results will suffer from speckles due to the SAR imaging mechanism just the same as matched filtering [11]. In this paper, the region-based features refer to the characteristics of distributed targets, such as uniformity, continuity, edge preservation, etc.

The graphics and properties of nonconvex penalties are more similar to those of the $L_{0}$-norm penalty compared with the $L_{1}$-norm penalty, therefore, several works have used nonconvex penalties in the model, including the minimax concave (MC) penalty $[12,13]$, the smoothly clipped absolute deviation (SCAD), the log sum penalty (LSP), and the $L_{q}$-norm $(0<q<1)$ penalty [14], to reduce the bias caused by $L_{1}$ regularization while ensuring the advantages of sparse signal processing. In addition to point-based features, region-based features are also important for many applications such as target classification and image segmentation, for which the TV-norm of the image amplitude is introduced into the SAR imaging model as a constraint. In [15], the authors propose a novel way of integrating both TV penalty and phase error into the cost function of the sparse SAR image reconstruction problem to improve the overall reconstruction quality. In [16], a TV regularization model for SAR image despeckling is introduced. Then [11] presents a solution to the complex SAR imaging problem within a constrained optimization formulation where the objective function includes a combination of the $L_{1}$-norm and the TV of the magnitude of the complex-valued reflectivity field. In [17], the authors analyze the performance of the compressive sensing-TV method in SAR imaging and autofocus. The above research shows that TV regularization can result in reconstructions with emphasized piecewise-constant features. Therefore, in a recently submitted contribution, we have proposed to linearly combine the nonconvex penalty and the TV-norm penalty as a compound regularizer in the imaging model, called nonconvex and TV regularization, which can not only reduce the bias caused by $L_{1}$ regularization but also enhance point-based and region-based features [18,19].

In this paper, based on the existing research work, we further optimize the algorithm and analyze the reconstruction performance for the nonconvex and TV regularization model. Firstly, we use the variable splitting scheme and modify the alternating direction method of multipliers (ADMM) in the algorithm, generating a novel algorithm to solve the nonconvex and TV regularization problem $[11,20]$. The algorithm can avoid the intermediate minimization problems becoming increasingly ill-conditioned, which appears in the quadratic penalty approach used in the previous paper [21]. Then, to quantitatively evaluate the reconstructed SAR image quality based on nonconvex and TV regularization, this paper analyzes the radiometric properties of sparse-signal-processing-based SAR imaging results, and introduces three indexes suitable for sparse SAR imaging [22,23]. Finally, as the first C-band multi-polarization spaceborne SAR in China, GF-3 plays an important role in providing high-quality data for scientific experiments, therefore, it has aroused our great interest to process data from the GF-3 satellite utilizing the nonconvex and TV regularization method. Experimental results and image quality analysis verify the effectiveness and advantages of the method: compared with $L_{1}$ regularization, the method can not only improve the reconstruction accuracy but also enhance region-based features represented by the radiometric resolution; compared with the matched filtering method, the method can suppress speckles as well as sidelobes and additive noise; compared with the method of speckle removal in the image domain including multilook processing [24,25], the method can process the raw echo data and generate SAR images after speckle reduction, without sacrificing the spatial resolution.

The rest of the paper is organized as follows. Section 2 constructs the imaging model, introduces the nonconvex and TV regularization method, and proposes the variable splitting-modified ADMM and sparse SAR imaging evaluation indexes. Section 3 shows the experimental results and image quality analysis based on GF-3 data processing. The discussions and conclusions are presented in Sections 4 and 5. 


\section{Materials and Methods}

\subsection{Sparse SAR Imaging Formation}

In SAR imaging, $\boldsymbol{X} \in \mathbb{C}^{N_{P} \times N_{Q}}$ denotes the complex-valued reflectivity matrix of the surveillance region and $Y \in \mathbb{C}^{N_{\eta} \times N_{\tau}}$ represents the sampled 2-D echo data. Then $\boldsymbol{x}=\operatorname{vec}(\boldsymbol{X}) \in \mathbb{C}^{N \times 1}$ with $N=N_{P} \times N_{Q}$, and $\boldsymbol{y}=\operatorname{vec}(\boldsymbol{Y}) \in \mathbb{C}^{M \times 1}$ with $M=N_{\eta} \times N_{\tau}$, where the vectorization operation $\operatorname{vec}(\cdot)$ stacks the columns one after the other. In this way, the relationship between the SAR measurements and the reflectivity of the scene to be reconstructed can be expressed as [2]:

$$
y=\Phi \alpha+n
$$

where $\boldsymbol{n} \in \mathbb{C}^{M \times 1}$ is the additive noise vector and $\boldsymbol{\Phi} \in \mathbb{C}^{M \times N}$ is the corresponding system measurement matrix between $y$ and $\boldsymbol{\alpha}$, as shown in Figure 1 .

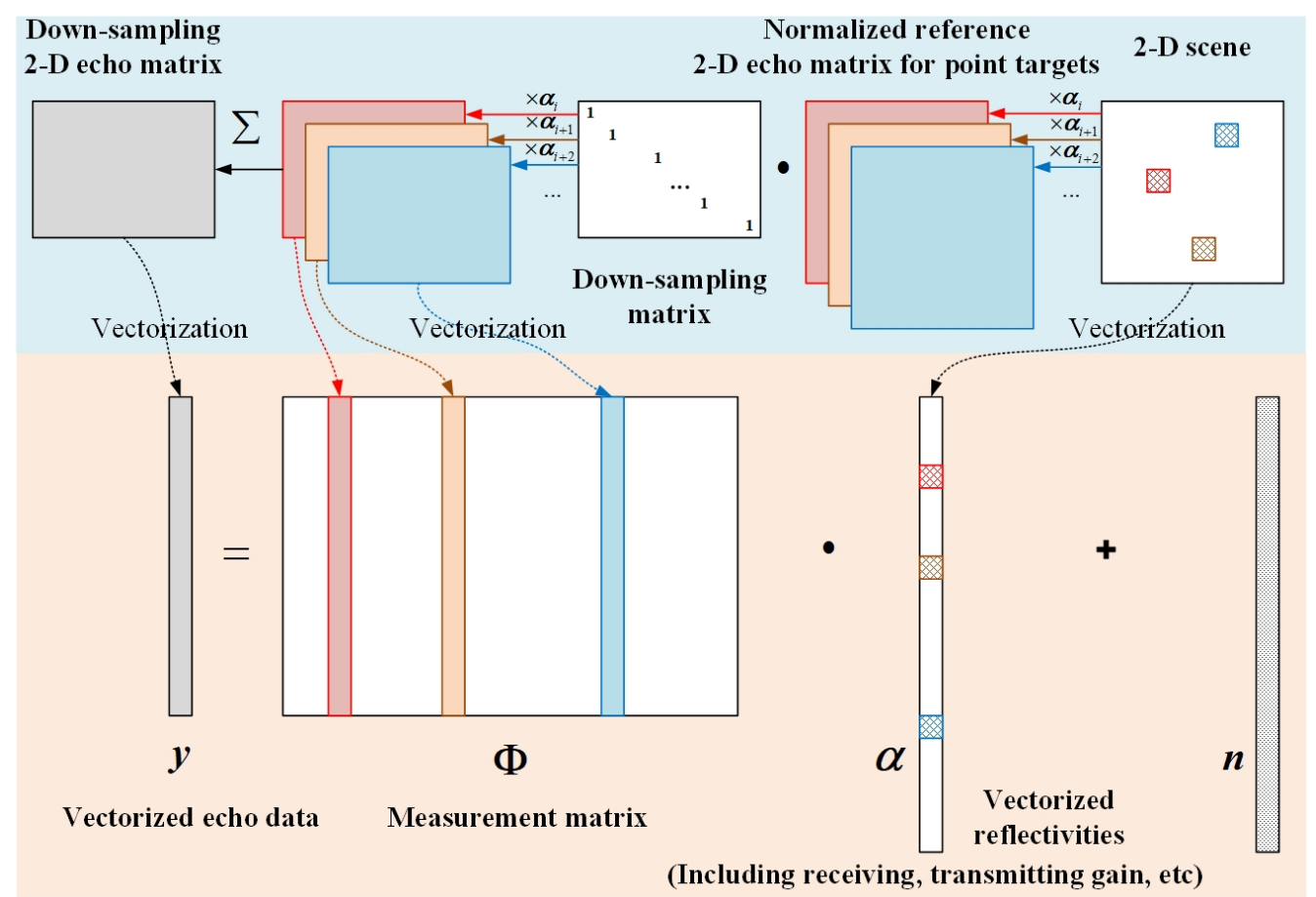

Figure 1. Schematic diagram of sparse SAR imaging model.

The SAR imaging process can be considered as a linear inverse problem (LIP), in which we try to solve $\alpha$ with $y$ and $\boldsymbol{\Phi}$. The most commonly used method for solving a LIP is to minimize the regularized linear least-squares cost function:

$$
\min _{\boldsymbol{\alpha}}\|\boldsymbol{y}-\boldsymbol{\Phi} \boldsymbol{\alpha}\|_{2}^{2}+\lambda p(\boldsymbol{\alpha})
$$

where $p(\cdot)$ is the penalty function or regularizer and $\lambda$ is the regularization parameter. If raw data $y$ are the down-sampled data, the model in (1) turns out to be an under-determined linear system, which has either no solution or infinitely many solutions. Downsampling can be divided into azimuth downsampling and range downsampling. If $Y \in \mathbb{C}^{N_{\eta} \times N_{\tau}}$ represents the full sampled 2-D echo data, the azimuth down-sampled data can be written as $\boldsymbol{D}_{a} \boldsymbol{Y}$ and the range down-sampled data are $\boldsymbol{Y} \boldsymbol{D}_{r}$, where the azimuth downsampling matrix $\boldsymbol{D}_{a}$ extracts some rows of the identity matrix $\boldsymbol{I} \in \mathbb{R}^{N_{\eta} \times N_{\tau}}$ as its own row elements and the range downsampling matrix $\boldsymbol{D}_{r}$ extracts some columns of the identity matrix $I \in \mathbb{R}^{N_{\eta} \times N_{\tau}}$ as its own column elements. For the ill-conditioned inverse problem, when $x$ is sparse enough and $\boldsymbol{\Phi}$ satisfies the condition of restricted isometry properties, we can obtain the unique solution of (1) via the $L_{1}$ regularization-based sparse SAR imaging method: 


$$
\min _{\boldsymbol{\alpha}}\|\boldsymbol{y}-\boldsymbol{\Phi} \boldsymbol{\alpha}\|_{2}^{2}+\lambda\|\boldsymbol{\alpha}\|_{1},\|\boldsymbol{\alpha}\|_{1}=\sum_{i=1}^{N}\left(\left|\alpha_{i}\right|\right)
$$

where $\alpha_{i}$ is the $i$ th element of $\alpha$. However, there are two main problems in the above imaging model: firstly, the $L_{1}$-norm penalty is convex and the analytic solution of the sparse signal reconstruction algorithm based on $L_{1}$ regularization is the biased estimation; secondly, $L_{1}$ regularization can only enhance point-based features in SAR images while ignoring region-based features, which means its reconstruction results will suffer from speckles due to the SAR imaging mechanism just the same as matched filtering. Therefore, it is necessary to find a suitable penalty function substituting for the $L_{1}$-norm penalty.

\subsection{Nonconvex and TV Regularization}

Given the above two problems, we linearly combine the nonconvex penalty and the TV-norm penalty as a compound regularizer, generating the following nonconvex and TV regularization model [18]:

$$
\min _{\boldsymbol{\alpha}}\|\boldsymbol{y}-\boldsymbol{\Phi} \boldsymbol{\alpha}\|_{2}^{2}+\lambda_{1} p_{N C}(\boldsymbol{\alpha})+\lambda_{2} p_{T V}(|\boldsymbol{\alpha}|)
$$

In the above formula, $\lambda_{1}$ and $\lambda_{2}$ are regularization parameters and $p_{N C}(\boldsymbol{\alpha})$ represents the nonconvex penalty of image vector $\boldsymbol{\alpha}$. In this paper, the minimax concave (MC) penalty is used as the representative of nonconvex penalties, as shown below [12,13]:

$$
p_{M C}(\boldsymbol{\alpha})=\sum_{i=1}^{N} p_{M C}\left(\alpha_{i}\right), \text { with } p_{M C}\left(\alpha_{i}\right)= \begin{cases}\left(\left|\alpha_{i}\right|-\frac{\alpha_{i}^{2}}{2 \theta}\right), & \left|\alpha_{i}\right| \leq \theta \\ \theta / 2, & \left|\alpha_{i}\right|>\theta\end{cases}
$$

And $p_{T V}(|\boldsymbol{\alpha}|)$ means the TV-norm penalty of image vector $\boldsymbol{\alpha}$. For two-dimensional scenes, the discrete isotropic TV-norm of $\boldsymbol{\alpha}$ is defined as follows [11]:

$$
p_{T V}(|\boldsymbol{\alpha}|)=\sum_{i, j}\left|\nabla\left(|\boldsymbol{A}|_{i, j}\right)\right|=\|\nabla(|\boldsymbol{A}|)\|_{1}
$$

where $A$ is the two-dimensional complex-valued matrix corresponding to image vector $\alpha$, the operator $|\cdot|$ represents element-wise magnitude calculation and $\nabla\left(|\boldsymbol{A}|_{i, j}\right)$ is the gradient vector of a pixel in the $i$ th row and $j$ th column, which is defined as:

$$
\begin{gathered}
\nabla\left(|\boldsymbol{A}|_{i, j}\right)=\left(D_{h}|\boldsymbol{A}|_{i, j}, D_{v}|A|_{i, j}\right),\left|\nabla\left(|A|_{i, j}\right)\right|=\sqrt{\left(D_{h}|A|_{i, j}\right)^{2}+\left(D_{v}|A|_{i, j}\right)^{2}} \\
D_{h}|A|_{i, j}=|A[i+1, j]|-|A[i, j]|, \\
D_{v}|A|_{i, j}=|A[i, j+1]|-|A[i, j]| .
\end{gathered}
$$

In the nonconvex and TV regularization-based sparse SAR imaging model, the nonconvex penalty, playing the role of sparsity inducing regularizer, can enhance point-based features as well as reducing the bias caused by $L_{1}$ regularization, and the TV-norm penalty will enhance region-based features, maintaining the continuity of the backscattering coefficient of distributed targets within a certain area. Therefore, the proposed method can improve the reconstruction accuracy of targets as well as protecting the reconstruction result from speckles, improving the radiometric resolution of SAR images.

\subsection{Variable Splitting and Modified ADMM}

For solving the nonconvex and TV regularization problem described in (4), we use the variable splitting scheme and modify the ADMM, generating a novel algorithm as shown in Figure 2. Firstly, under the guidance of the variable splitting scheme, we convert the unconstrained optimization problem (4) to the following equivalent constrained optimization problem [11]: 


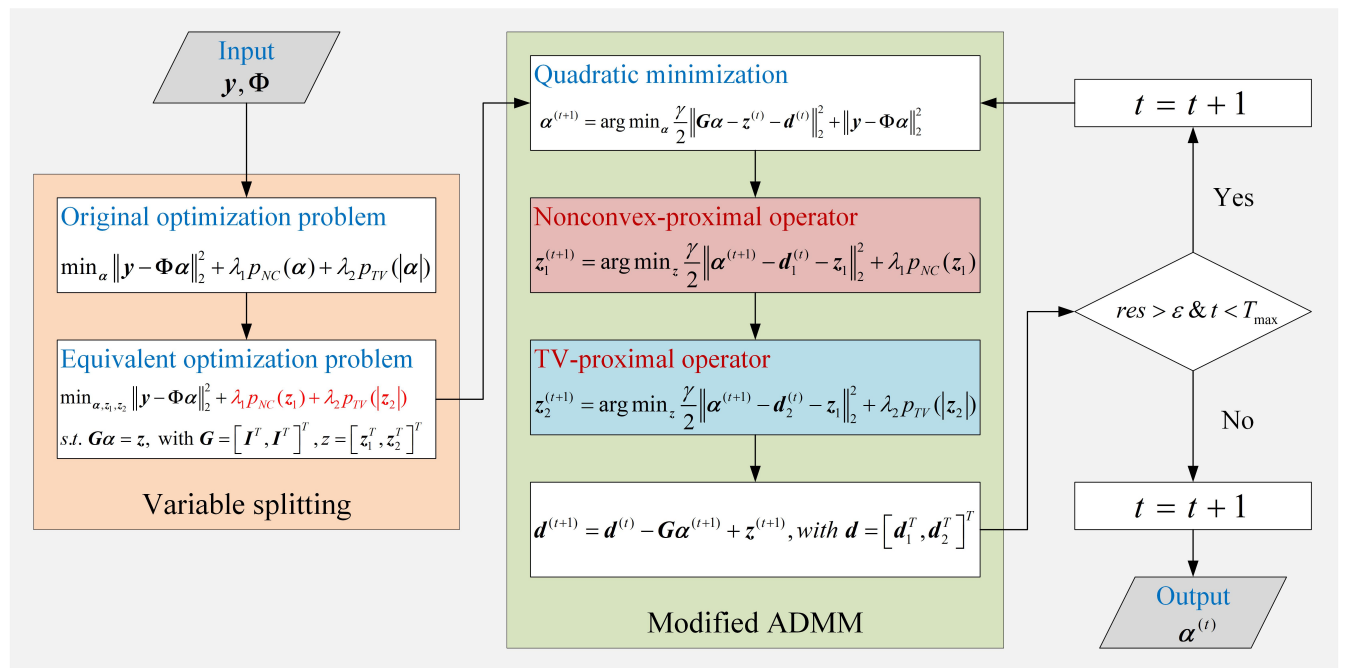

Figure 2. Flow chart of the proposed algorithm combining variable splitting and modified ADMM.

$$
\begin{aligned}
& \min _{\boldsymbol{\alpha}, z_{1}, z_{2}}\|\boldsymbol{y}-\boldsymbol{\Phi} \boldsymbol{\alpha}\|_{2}^{2}+\lambda_{1} p_{M C}\left(\boldsymbol{z}_{\mathbf{1}}\right)+\lambda_{2} p_{T V}\left(\left|z_{\mathbf{2}}\right|\right) \\
& \text { s.t. } \boldsymbol{G} \boldsymbol{\alpha}=\boldsymbol{z} \text {, with } \boldsymbol{G}=\left[\boldsymbol{I}^{T}, \boldsymbol{I}^{T}\right]^{T}, \boldsymbol{z}=\left[\boldsymbol{z}_{\mathbf{1}}{ }^{T}, \boldsymbol{z}_{\mathbf{2}}{ }^{T}\right]^{T}
\end{aligned}
$$

where $z_{1}, z_{2}$ are two auxiliary variables and $I$ is the identity matrix. Then, according to the principle of ADMM, the above optimization problem can be solved via alternating minimization as below:

$$
\begin{aligned}
s u b-p 1: \boldsymbol{\alpha}^{(t+1)} & =\operatorname{argmin}_{\boldsymbol{\alpha}} \frac{\gamma}{2}\left\|\boldsymbol{G} \boldsymbol{\alpha}-\boldsymbol{z}^{(t)}-\boldsymbol{d}^{(t)}\right\|_{2}^{2}+\|\boldsymbol{y}-\boldsymbol{\Phi} \boldsymbol{\alpha}\|_{2}^{2}, \\
s u b-p 2: z_{1}^{(t+1)} & =\operatorname{argmin}_{z} \frac{\gamma}{2}\left(\left\|\boldsymbol{\alpha}^{(t+1)}-\boldsymbol{d}_{1}^{(t)}-z_{1}\right\|_{2}^{2}\right)+\lambda_{1} p_{M C}\left(z_{1}\right), \\
s u b-p 3: z_{2}^{(t+1)} & =\operatorname{argmin}_{z} \frac{\gamma}{2}\left(\left\|\boldsymbol{\alpha}^{(t+1)}-\boldsymbol{d}_{2}^{(t)}-z_{2}\right\|_{2}^{2}\right)+\lambda_{2} p_{T V}\left(\left|z_{2}\right|\right), \\
\boldsymbol{d}^{(t+1)} & =\boldsymbol{d}^{(t)}-\boldsymbol{G} \boldsymbol{\alpha}^{(t+1)}+\boldsymbol{z}^{(t+1)}, \text { with } \boldsymbol{d}=\left[\boldsymbol{d}_{\mathbf{1}}{ }^{T}, \boldsymbol{d}_{\mathbf{2}}{ }^{T}\right]^{T} .
\end{aligned}
$$

In the above optimization problems, $d$ is a variant of the Lagrange multiplier vector and $\gamma \geq 0$ is called the augmented Lagrangian penalty parameter.

For ease of understanding, the objective function of sub-problem 1 in (11) can be written as a strictly convex quadratic function:

$$
\boldsymbol{\alpha}^{(t+1)}=\operatorname{argmin}_{\boldsymbol{\alpha}}\left\|\left[\begin{array}{c}
\sqrt{\frac{\gamma}{2}} \boldsymbol{G} \\
\boldsymbol{\Phi}
\end{array}\right] \boldsymbol{\alpha}-\left[\begin{array}{c}
\sqrt{\frac{\gamma}{2}}\left(\boldsymbol{z}^{(t)}+\boldsymbol{d}^{(t)}\right) \\
\boldsymbol{y}
\end{array}\right]\right\|_{2}^{2}=\operatorname{argmin}_{\boldsymbol{\alpha}}\|\boldsymbol{H} \boldsymbol{\alpha}-\boldsymbol{\beta}\|_{2}^{2},
$$

which leads to the following optimal solution according to the principle of gradient descent, and $\frac{1}{L}$ is the step size of the iteration:

$$
\boldsymbol{\alpha}^{(t+1)}=\boldsymbol{\alpha}^{(t)}+\frac{1}{L} \boldsymbol{H}^{H}\left(\boldsymbol{\beta}-\boldsymbol{H} \boldsymbol{\alpha}^{(t)}\right)=\boldsymbol{\alpha}^{(t)}+\frac{1}{L}\left[\frac{\gamma}{2} \sum_{i=1}^{2}\left(\boldsymbol{d}_{i}^{(t)}+z_{i}^{(t)}\right)-\gamma \boldsymbol{\alpha}^{(t)}+\boldsymbol{\Phi}^{H}\left(\boldsymbol{y}-\boldsymbol{\Phi} \boldsymbol{\alpha}^{(t)}\right)\right] \text {. }
$$

Sub-problem 2 in (11) is a nonconvex optimization problem, which cannot be solved via the traditional ADMM. Therefore, we modify the ADMM by borrowing the idea of the general iterative shrinkage-thresholding algorithm, in which the author made some constraints on the nonconvex penalty so that the algorithm could solve nonconvex optimization problems [14]. According to the reference, when the nonconvex penalty $p_{N C}(\boldsymbol{\alpha})$ satisfies the following three assumptions:

- $\quad p_{N C}(\boldsymbol{\alpha})$ is a continuous function that is possibly nonsmooth and nonconvex, and can be rewritten as the difference of two convex functions; 
- $\quad p_{N C}(\boldsymbol{\alpha})$ can be written as $p_{N C}(\boldsymbol{\alpha})=\sum_{i=1}^{N} p_{N C}\left(\alpha_{i}\right)$;

- the objective function is lower-bounded;

there exists a simple closed-form solution to sub-problem 2 in (11). The MC penalty used in this paper obviously meets the above conditions, and the closed-form solution corresponding to the MC penalty can be expressed as $(\theta>1)$ :

$$
\begin{aligned}
& z_{1}^{(t+1)}=\Psi_{M C}\left(\boldsymbol{\alpha}^{(t+1)}-\boldsymbol{d}_{1}^{(t)}, \frac{\lambda_{1}}{\gamma}\right), \text { with } \\
& \Psi_{M C}\left(\alpha_{i}, \lambda\right)= \begin{cases}0, & \left|\alpha_{i}\right|<\lambda \\
\theta \lambda \frac{\left|\alpha_{i}\right|-\lambda}{\theta \lambda-\lambda} \operatorname{sign}\left(\alpha_{i}\right), & \lambda \leq\left|\alpha_{i}\right| \leq \theta \lambda \\
\alpha_{i}, & \left|\alpha_{i}\right|>\theta \lambda .\end{cases}
\end{aligned}
$$

Sub-problem 3 in (11) is the TV regularization problem and we can obtain its closedform solution using the Chambolle algorithm:

$$
z_{2}^{(t+1)}=\Psi_{T V}\left(\boldsymbol{\alpha}^{(t+1)}-\boldsymbol{d}_{2}^{(t)}, \frac{\lambda_{2}}{\gamma}\right),
$$

for more details of the Chambolle algorithm, please refer to the related literature [26].

There still exists a problem in the above algorithm: it has to store and process the large-scale measurement matrix, which would bring huge memory and computational cost to SAR imaging. Thus, we adopt the azimuth-range decouple scheme in the real data processing [27], in which the measurement matrix $\boldsymbol{\Phi}$ and its Hermitian transpose $\boldsymbol{\Phi}^{H}$ can be approximately replaced by the raw data generation operator $\mathrm{Ge}(\cdot)$ and the imaging operator $\operatorname{Im}(\cdot)$. Applying the azimuth-range decouple scheme to iterations, we can obtain the optimal solution of the problem (11) through the following iterative algorithm, as shown in Algorithm 1. In practical applications, the value of $\lambda_{1}$ needs to be determined according to the sparsity of the scene, and the value of $\lambda_{2}$ needs to be determined according to the uniformity of distributed targets in the scene.

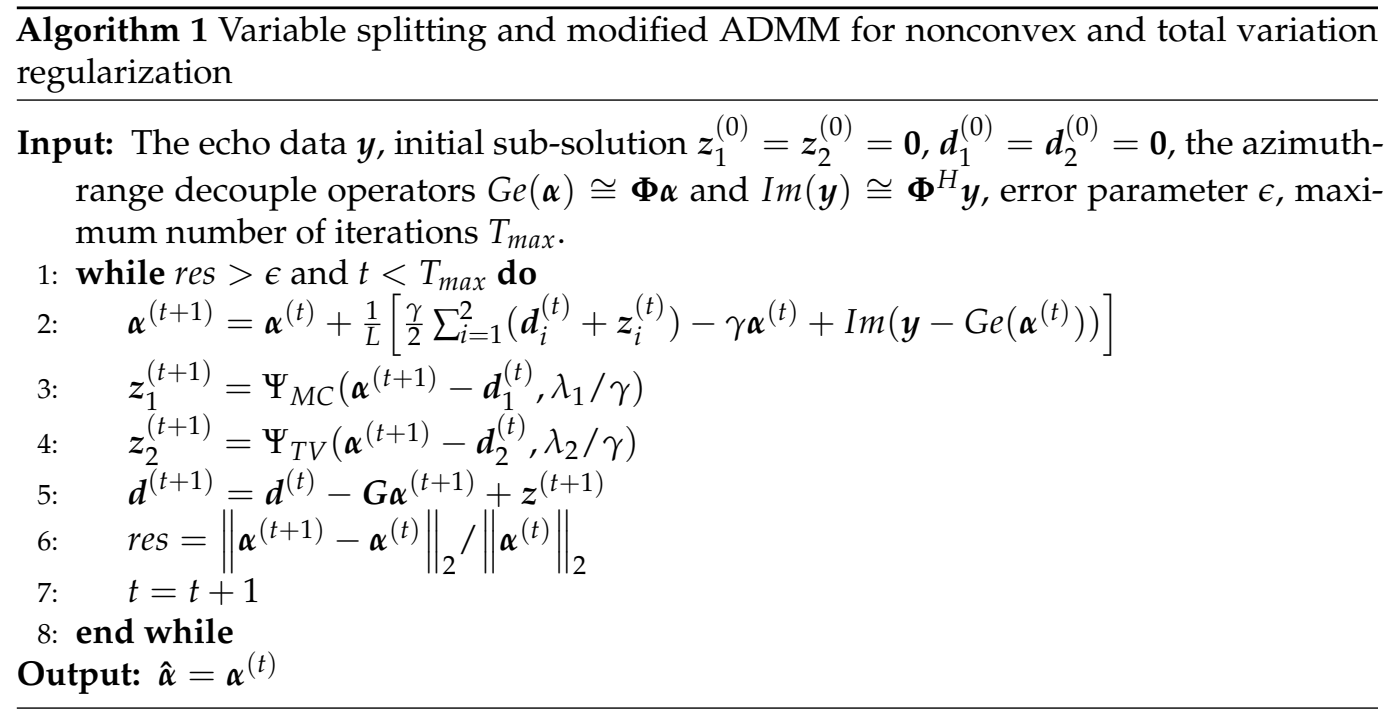

\subsection{Sparse SAR Imaging Evaluation Index}

- Physical Significance of Sparse SAR Imaging Results

As shown in Equation (1) and Figure 1, the observation model of the SAR can be regarded as a linear system. Likewise, the imaging results of the matched filtering method can be written as [28]:

$$
\hat{\boldsymbol{\alpha}}_{M F}=\boldsymbol{\Phi}^{H} \boldsymbol{y}=\boldsymbol{\Phi}^{H} \boldsymbol{\Phi} \cdot \boldsymbol{\alpha}+\boldsymbol{n}^{\prime}=C_{2} \cdot\left(C_{1} \sqrt{\sigma} e^{j \phi(\boldsymbol{\alpha})}\right)+\boldsymbol{n}^{\prime} .
$$


In the above expression, the weighted complex reflectivity $\alpha$ not only represents the radar cross-section (RCS) of the target $(\sigma)$, but also includes the energy weighting of the radar system $\left(C_{1}\right)$, as shown in Figure 3:

$$
\left|C_{1}\right|^{2}=\frac{P_{t} G^{2} \lambda^{2}}{(4 \pi)^{3} R^{4} L} .
$$

where $P_{t}$ is the peak transmitted power, $G$ is the one-way power gain of the antenna pattern, $\mathrm{R}$ is the distance between radar and target, and $L$ allows for system losses. In addition, $C_{2}=G_{P R} \times G_{P A}$ is the processing gain due to the range compression $\left(G_{P R}\right)$ and azimuth compression $\left(G_{P A}\right)$, corresponding to $\boldsymbol{\Phi}^{H} \boldsymbol{\Phi}$ in Equation (16). $\boldsymbol{n}^{\prime}$ is additive noise, and it also contains ambiguity under the condition of downsampling.

The theoretical result of measurement-matrix-based sparse SAR imaging can be expressed as:

$$
\hat{\boldsymbol{\alpha}}_{S_{M}}=\alpha=C_{1} \sqrt{\sigma} e^{j \phi(\alpha)},
$$

while the azimuth-range decouple-operators-based sparse SAR imaging results have the following expression:

$$
\hat{\boldsymbol{\alpha}}_{S_{O}}=\boldsymbol{\Phi}^{H} \boldsymbol{\Phi} \cdot \boldsymbol{\alpha}=C_{2} \cdot\left(C_{1} \sqrt{\sigma} e^{j \phi(\boldsymbol{\alpha})}\right) .
$$

Therefore, we can think of the azimuth-range decouple-operators-based sparse SAR imaging results $\hat{\boldsymbol{\alpha}}_{S_{O}}$ as the noiseless version of the matched-filtering-method-based SAR imaging results $\hat{\boldsymbol{\alpha}}_{M F}$. It should be noted that the above analysis is carried out for the point target. For distributed targets, we need to replace $\sigma$ with $\sigma=\sigma_{0} \rho_{g} \rho_{a}$ in Equations (16)-(19), where $\rho_{g}$ denotes the ground resolution and $\rho_{a}$ denotes the azimuth resolution.

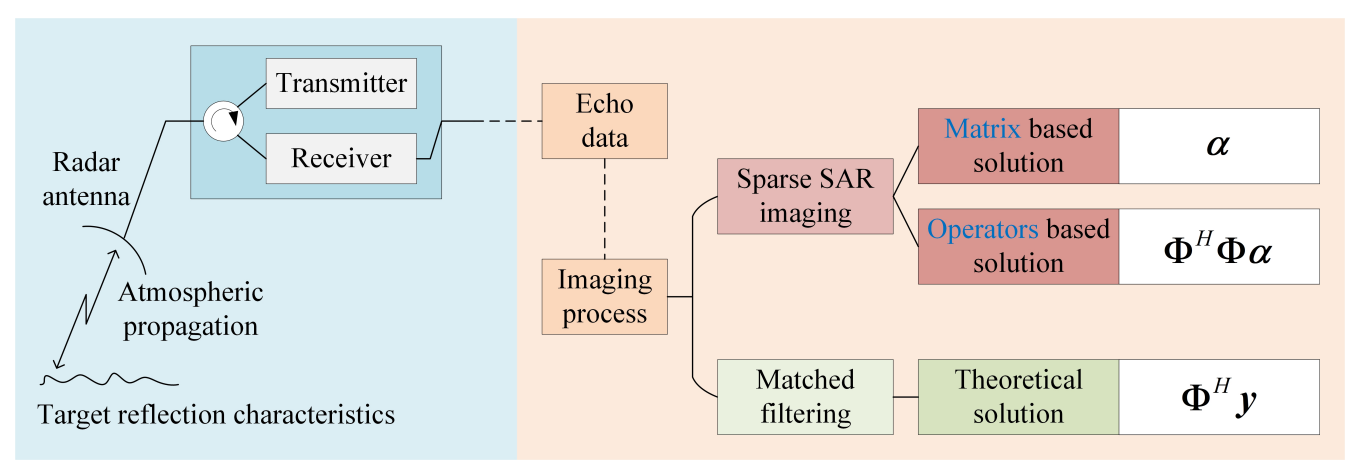

Figure 3. Various factors affecting SAR imaging results.

- Radiometric Accuracy

Through the above analysis, we can see that in the ideal case, for targets in the scene, the reconstruction results of azimuth-range decouple-operators-based sparse SAR imaging $\left(\hat{\boldsymbol{\alpha}}_{S_{O}, T a r}\right)$ should be almost the same as that of the matched filtering method $\left(\hat{\boldsymbol{\alpha}}_{M F, T a r}\right)$. However, some sparse SAR imaging methods cannot reconstruct targets accurately due to the bias effects caused by $L_{1}$ regularization. Here, we take the reconstruction result of the matched filtering method as the standard to measure the reconstruction accuracy of azimuth-range decouple-operatorsbased sparse SAR imaging. The more similar $\hat{\boldsymbol{\alpha}}_{S_{O}, T a r}$ is to $\hat{\boldsymbol{\alpha}}_{M F, T a r}$, the higher the reconstruction accuracy of azimuth-range decouple-operatorsbased sparse SAR imaging is, and the more accurate the estimation of RCS is, which means higher radiometric accuracy. To quantitatively analyze the reconstruction accuracy, we define relative bias (RB) as the evaluation criteria:

$$
\begin{aligned}
& R B_{p}=|\hat{\alpha}-\alpha| /|\alpha|, \\
& R B_{d}=\left|\hat{\alpha_{\mu}}-\alpha_{\mu}\right| /\left|\alpha_{\mu}\right|
\end{aligned}
$$


where $R B_{p}$ means the relative bias of point targets and $R B_{d}$ is the relative bias of distributed targets. Correspondingly, $\alpha$ is the imaging result of the point target based on the matched filtering method, which is regarded as the ground truth here, and $\hat{\alpha}$ is the estimation of $\alpha$ using the azimuth-range decouple-operators-based sparse SAR imaging method. Because the noise of coherent speckle exists in the SAR image, we use the mean value of the reconstruction results $\left(\alpha_{\mu}\right.$-matched filtering, $\hat{\alpha_{\mu}}$-sparse signal processing $)$ in the definition of relative bias for distributed targets here.

\section{- $\quad$ Radiometric Resolution}

In addition to impulse response properties, image quality assessment must consider the response of the system to distributed targets. Generally, the equivalent number of looks (ENL) and radiometric resolution (RR) are used to measure the reconstruction quality of distributed targets. The definition of ENL is derived from multilook processing, which can be operated based on an intensity or amplitude SAR image. According to the relevant reference [29], amplitude-averaging-based ENL has the following expression:

$$
E N L=0.5227^{2} \times \frac{\mu(A)^{2}}{\sigma(A)^{2}}
$$

where $\mu(A)$ is the mean of amplitude SAR images and $\sigma(A)$ is the standard deviation. Furthermore, we can get the definition of radiometric resolution as below [22,23]:

$$
\gamma(d B)=10 \cdot \lg \left(1+\frac{1}{\sqrt{E N L}}\right)=10 \cdot \lg \left(1+\frac{1}{0.5227} \times \frac{\sigma(A)}{\mu(A)}\right) .
$$

As nonconvex and TV-regularization-based sparse SAR imaging generates the amplitude SAR image, which is the same as operating multilook processing based on the amplitude SAR image, it is reasonable for us to use the above definition to evaluate the reconstruction quality of distributed targets.

- Spatial Resolution

Main lobe width (MLW), also called the spatial resolution, is measured by the distance between the points with intensities $3 \mathrm{~dB}$ below the maximum intensity of the main lobe peak in the azimuth and range direction, standing for the distance between two objects on the ground to the extent that they can be distinct or separate. In the radar image obtained by matched filtering, the system impulse response of the ideal point target is the $\operatorname{sinc}(\cdot)$ function, so we can use MLW to measure the spatial resolution. However, the ideal reconstruction result of the point target in measurement-matrix-based sparse microwave imaging is the impulse function $\delta(\cdot)$, so it is no longer suitable to calculate MLW to measure spatial resolution [2]. Fortunately, as shown in Equation (19), the azimuth-range decouple-operators-based sparse SAR imaging results $\hat{\boldsymbol{\alpha}}_{S_{O}}$ can be regarded as the noiseless version of the matched-filtering-method-based SAR imaging results $\hat{\boldsymbol{\alpha}}_{M F}$, which means the system impulse response of the ideal point target is approximately a $\operatorname{sinc}(\cdot)$ function, so we can calculate MLW to measure the spatial resolution of $\hat{\boldsymbol{\alpha}}_{S_{O}}$.

\section{Results}

\subsection{GF-3 SAR Data Description}

GF-3 is the first C-band remote sensing satellite with quad-polarization and multiangle capability in the civilian field of China, and operates in 12 imaging modes with spatial resolution up to $1 \mathrm{~m}$. In this paper, we select two pieces of stripmap mode GF-3 SAR raw echo data for experiments.

Data 1: To verify the ability of nonconvex and TV regularization in improving reconstruction accuracy, we choose an area that includes many farmland regions with different backscattering coefficients as shown in Figure 4a. Considering that the backscattering coefficient of the farmland region is relatively uniform, we can think of them as distributed targets. In SAR images, the gray scale of farmland regions is different, which indicates the 
difference in backscattering coefficients between them. This scene can be used to analyze the reconstruction accuracy of distributed targets with different backscattering coefficients, and to study the maintenance of the relative relationship between them.

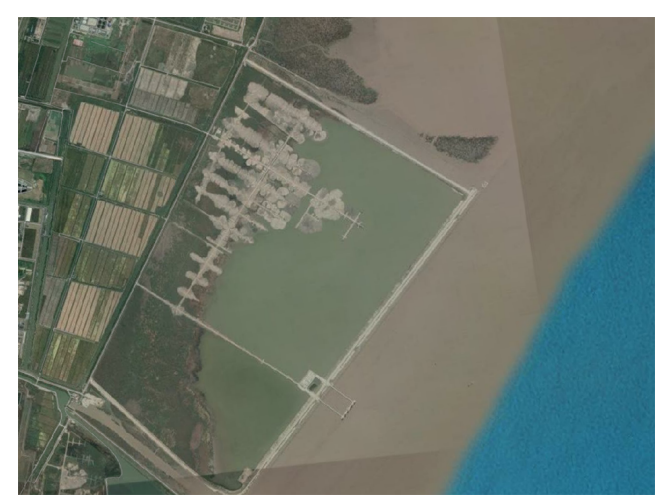

(a)

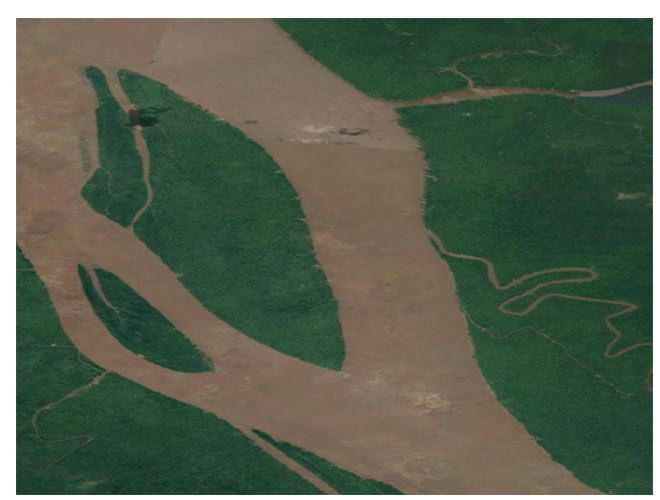

(b)

Figure 4. Optical remote sensing image of experimental areas (provided by Google Earth). (a) Scene 1 corresponding to data 1 . (b) Scene 2 corresponding to data 2 . The size of scene 1 is $8673 \mathrm{~m}$ (vertical direction) $\times 8867 \mathrm{~m}$ (horizontal direction). The size of scene 2 is $19,228 \mathrm{~m}$ (vertical direction) $\times$ $4935 \mathrm{~m}$ (horizontal direction).

Data 2: The second piece of data was collected in the Amazon rainforest area, which has a quite homogeneous backscattering coefficient around a wide area, as shown in Figure $4 \mathrm{~b}$. The rainforest area in the scene is divided into several pieces by the river, but they still have almost the same backscattering coefficient; the scene also shows a certain degree of sparsity. Therefore, we will use it to explore the performance of nonconvex and TV regularization in improving radiometric resolution. In addition, we have added a piece of simulated echo data of a point target to the raw SAR data, for verifying the ability of imaging methods to maintain point-based features when enhancing region-based features.

\subsection{Data Processing and Image Quality Assessment}

\section{- Experiment 1}

Firstly, we utilize the chirp scaling algorithm (CSA) to process the data 1 [30]. As shown in Figure 5a, there are obvious speckles in farmland regions. The simplest approach to despeckling is to average the amplitude over several pixels within a window centered on a specific pixel, known as multilook (ML) processing [24]. The result of applying this method to the original image in Figure 5 a, using a 5 by 5 window, is shown in Figure $5 \mathrm{~b}$. Secondly, $L_{1}$ regularization is used to process the data, with results shown in Figure 5c. Compared with Figure 5a,b, the figure has less noise and clutter. However, as $L_{1}$ regularization-based sparse SAR imaging methods just aim at enhancing point-based features, their reconstruction results also suffer from speckles due to the SAR imaging mechanism just the same as matched filtering. Finally, we reconstruct the experimental scene via $L_{1}$ and TV regularization and nonconvex and TV regularization. As shown in Figure $5 \mathrm{~d}$,e, the two methods can not only suppress noise and clutter but also maintain the uniformity and continuity of distributed targets. All the images in Figure 5 are plotted with the same color map. 


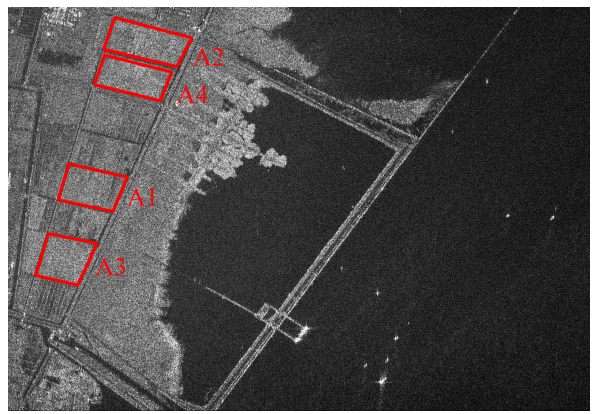

(a)

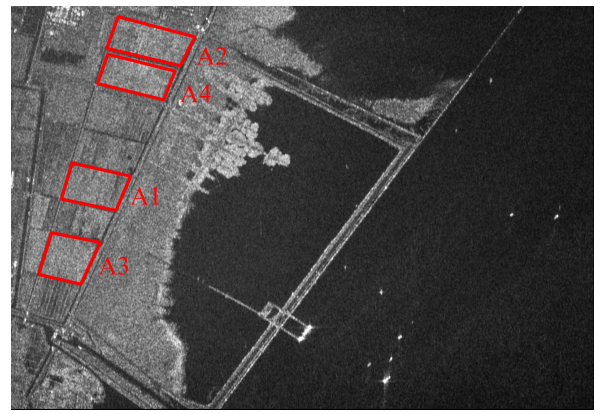

(b)

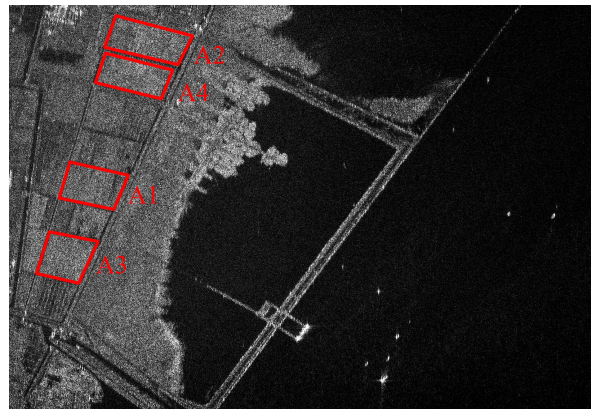

(c)

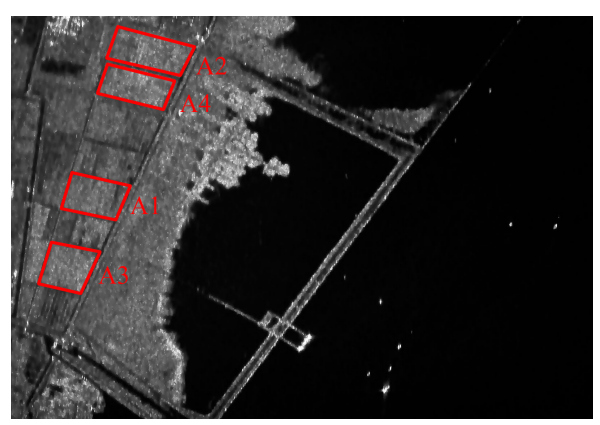

(d)

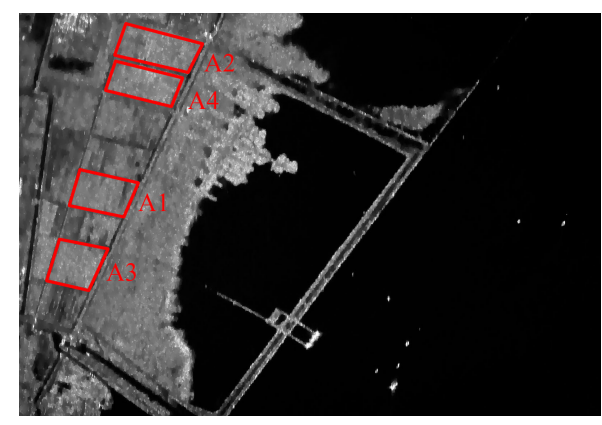

(e)

Figure 5. Experimental results for data 1 under full sampling condition. (a) Chirp scaling. (b) CSA + multilook processing using a 5 by 5 window. (c) $L_{1}$ regularization. (d) $L_{1}$ and TV regularization. (e) Nonconvex and TV regularization. The vertical direction of the image is the azimuth direction, and the horizontal direction is the range direction. SAR data 1 occupies 1404 (azimuth) $\times 3941$ (range) sampling points, and the size of the corresponding scene is $8673 \mathrm{~m}$ (azimuth) $\times$ $8867 \mathrm{~m}$ (range). All the images are plotted with the same color map.

For quantitative analysis of reconstruction accuracy, we select four areas as experimental distributed targets, which are surrounded by the red rectangle and labeled with A1/A2/A3/A4 in Figure 5a-e, and calculate the relative bias (RB) of the four targets according to Equation (14). Table 1 shows that the average reconstruction $R B$ of nonconvex and TV regularization is $2.42 \%$ when we regard the result of the matched filtering method as the ground truth, while the average reconstruction $R B$ s of $L_{1}$ regularization and $L_{1}$ and TV regularization are $23.4 \%$ and $32.9 \%$ respectively. The statistical results show that nonconvex and TV regularization has higher reconstruction accuracy than $L_{1}$ regularization and $L_{1}$ and TV regularization. Furthermore, we also calculate the ratio of the mean amplitude of target A2, A3, A4 to target A1; the results in Table 1 can demonstrate that nonconvex and TV regularization can better maintain the relative relationship between different targets compared with $L_{1}$ regularization and $L_{1}$ and TV regularization.

Table 1. The relative bias and relative relationship of four areas after relative normalization in experiment 1 for the five methods.

\begin{tabular}{|c|c|c|c|c|c|c|c|c|c|c|c|}
\hline \multirow{2}{*}{ Method } & \multicolumn{2}{|c|}{ A1 } & \multicolumn{3}{|c|}{ A2 } & \multicolumn{3}{|c|}{ A3 } & \multicolumn{3}{|c|}{ A4 } \\
\hline & $\mu_{A 1}$ & $R B_{A 1}$ & $\mu_{A 2}$ & $R B_{A 2}$ & $\mu_{A 2} / \mu_{A 1}$ & $\mu_{A 3}$ & $R B_{A 3}$ & $\mu_{A 3} / \mu_{A 1}$ & $\mu_{A 4}$ & $R B_{A 4}$ & $\mu_{A 4} / \mu_{A 1}$ \\
\hline CSA & 10.912 & / & 11.383 & / & 1.0432 & 11.664 & / & 1.0689 & 12.374 & / & 1.1340 \\
\hline $\mathrm{CSA}+\mathrm{ML}$ & 10.906 & / & 11.375 & / & 1.0430 & 11.671 & / & 1.0701 & 12.367 & / & 1.1340 \\
\hline$L_{1}$ & 8.2309 & 0.2457 & 8.6820 & 0.2373 & 1.0548 & 8.9710 & 0.2309 & 1.0899 & 9.6036 & 0.2239 & 1.1668 \\
\hline$L_{1}$ and TV & 7.1261 & 0.3469 & 7.5889 & 0.3333 & 1.0649 & 7.8861 & 0.3239 & 1.1067 & 8.5145 & 0.3119 & 1.1948 \\
\hline $\mathrm{MC}$ and TV & 10.557 & 0.0325 & 11.077 & 0.0269 & 1.0493 & 11.447 & 0.0186 & 1.0843 & 12.141 & 0.0188 & 1.1500 \\
\hline
\end{tabular}




\section{- $\quad$ Experiment 2}

In this part, we process data 2 using the same five methods as above: CSA, CSA + multilook processing using a 9 by 9 window, $L_{1}$ regularization, $L_{1}$ and TV regularization, and the proposed nonconvex and TV regularization. As shown in Figure 6, the latter three sparse SAR imaging methods can obviously suppress clutter and noise, meanwhile, nonconvex and TV regularization and $L_{1}$ and TV regularization can also maintain the uniformity and continuity of the backscattering coefficient in rainforest areas. All the images in Figure 6 are plotted with the same color map. To evaluate the radiometric resolution of the reconstructed SAR image, as in experiment 1 , we also select four areas as experimental distributed targets, which are surrounded by the red rectangle and labeled with F1/F2/F3/F4 in Figure 6. In addition, there is a simulated point target in the scene, surrounded by the red dotted circle and labeled with $\mathrm{P} 1$, for assessment of the spatial resolution.

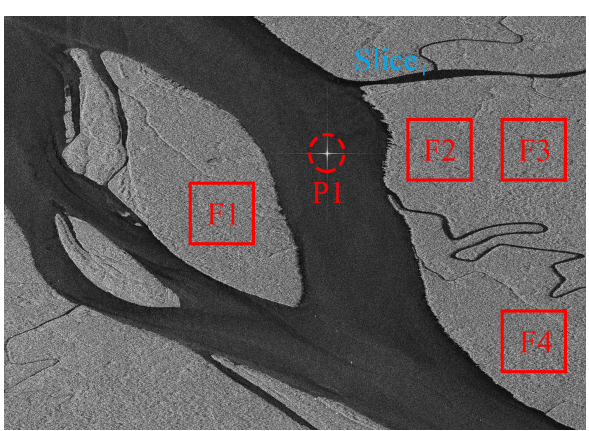

(a)

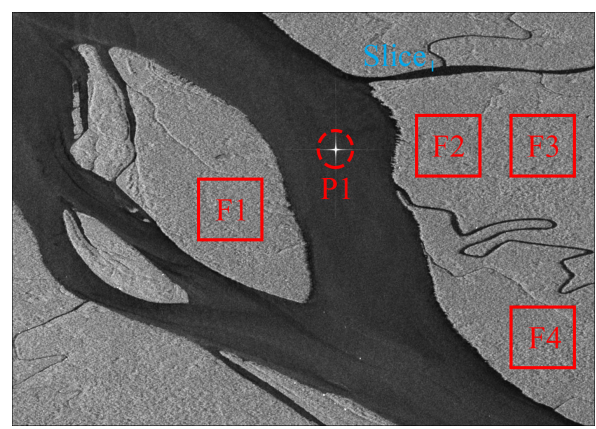

(b)

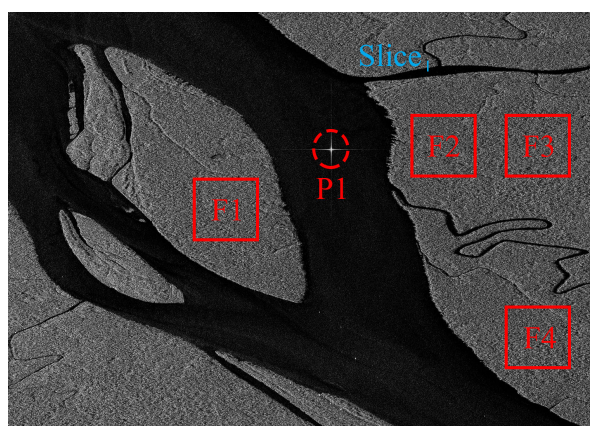

(c)

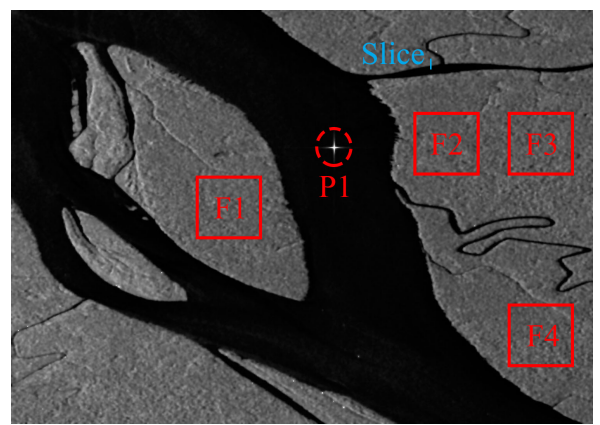

(d)

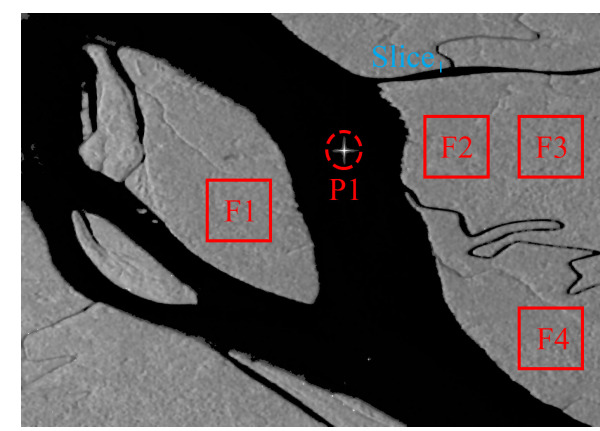

(e)

Figure 6. Experimental results for data 2 under full sampling condition. (a) Chirp scaling. (b) CSA + multilook processing using a 9 by 9 window. (c) $L_{1}$ regularization. (d) $L_{1}$ and TV regularization. (e) Nonconvex and TV regularization. The vertical direction of the image is the azimuth direction, and the horizontal direction is the range direction. SAR data 2 occupies 3115 (azimuth) $\times 4387$ (range) sampling points, and the size of the corresponding scene is 19,228 $\mathrm{m}($ azimuth) $\times$ $4935 \mathrm{~m}$ (range). All the images are plotted with the same color map.

Then we calculate the mean and variance of the four distributed targets for getting ENL and radiometric resolution. As shown in Table 2, the average radiometric resolution $(\gamma(\mathrm{dB}))$ of reconstruction results based on the matched filtering method, $L_{1}$ regularization and nonconvex and TV regularization are $3.13 \mathrm{~dB}, 4.25 \mathrm{~dB}$ and $0.46 \mathrm{~dB}$, respectively. The lower the value of $\gamma$, the higher the radiometric resolution, therefore, it can be further calculated that nonconvex and TV regularization can improve the radiometric resolution by an average of $85.28 \%, 89.19 \%$ compared with the matched filtering method and $L_{1}$ regularization, which verifies the effectiveness of nonconvex and TV regularization in improving the radiometric resolution on the one hand, and that the bias effect caused by $L_{1}$ regularization has a certain degree of adverse impact on the radiometric resolution on the other hand. 
Table 2. The radiometric resolution of four distributed targets in experiment 2 for the five methods.

\begin{tabular}{cccccc}
\hline Method & $\boldsymbol{\mu}$ & $\sigma^{\mathbf{2}}$ & ENL & $\gamma(\mathrm{dB})$ & Target \\
\hline CSA & 2.3668 & 1.7217 & 0.8889 & 3.1401 & \\
CSA + ML & 2.3661 & 0.1104 & 13.8518 & 1.0335 & F1 \\
$L_{1}$ & 1.4625 & 1.5837 & 0.3690 & 4.2263 & \\
$L_{1}$ and TV & 1.4029 & 0.0255 & 21.080 & 0.8558 & \\
MC and TV & $\mathbf{2 . 3 6 6 5}$ & $\mathbf{0 . 0 1 9 3}$ & $\mathbf{7 9 . 3 4 0}$ & $\mathbf{0 . 4 6 2 1}$ & \\
\hline CSA & 2.2768 & 1.5575 & 0.9093 & 3.1148 & \\
CSA + ML & 2.2763 & 0.0871 & 16.2510 & 0.9624 & F2 \\
$L_{1}$ & 1.3752 & 1.4180 & 0.3644 & 4.2434 & \\
$L_{1}$ and TV & 1.3124 & 0.0217 & 21.667 & 0.8452 & \\
MC and TV & $\mathbf{2 . 2 7 5 9}$ & $\mathbf{0 . 0 1 7 0}$ & $\mathbf{8 3 . 4 0 3}$ & $\mathbf{0 . 4 5 1 3}$ & \\
\hline CSA & 2.2863 & 1.5989 & 0.8932 & 3.1347 & \\
CSA + ML & 2.2864 & 0.1027 & 13.9013 & 1.0319 & \\
$L_{1}$ & 1.3844 & 1.4597 & 0.3587 & 4.2646 & \\
$L_{1}$ and TV & 1.3218 & 0.0244 & 19.548 & 0.8855 & \\
MC and TV & $\mathbf{2 . 2 8 5 3}$ & $\mathbf{0 . 0 1 8 3}$ & $\mathbf{7 8 . 1 6 2}$ & $\mathbf{0 . 4 6 5 4}$ & \\
\hline CSA & 2.2835 & 1.6114 & 0.8941 & 3.1461 & \\
CSA + ML & 2.2834 & 0.1056 & 13.4943 & 1.0456 & \\
$L_{1}$ & 1.3823 & 1.4701 & 0.3551 & 4.2782 & \\
$L_{1}$ and TV & 1.3192 & 0.0240 & 19.774 & 0.8809 & \\
MC and TV & $\mathbf{2 . 2 8 2 7}$ & $\mathbf{0 . 0 1 8 3}$ & $\mathbf{7 7 . 9 5 9}$ & $\mathbf{0 . 4 6 6 0}$ & \\
\hline
\end{tabular}

Finally, we slice the edge of the rainforest marked with a blue line in Figure 6 and the simulated point along the azimuth direction to compare the spatial resolution between the multilook processing and nonconvex and TV regularization. Figure 7a shows that the edge in the imaging results of nonconvex and TV regularization is sharper than that of the multilook processing, which means that nonconvex and TV regularization has better edge-preserving ability than the multilook processing, and further proves the ability of nonconvex and TV regularization to maintain the spatial resolution. In Figure $7 \mathrm{~b}$, it is obvious that nonconvex and TV regularization has nearly the same spatial resolution as chirp scaling while the multilook processing widens the main lobe, which also proves that nonconvex and TV regularization can maintain the same spatial resolution as the matched filtering method.

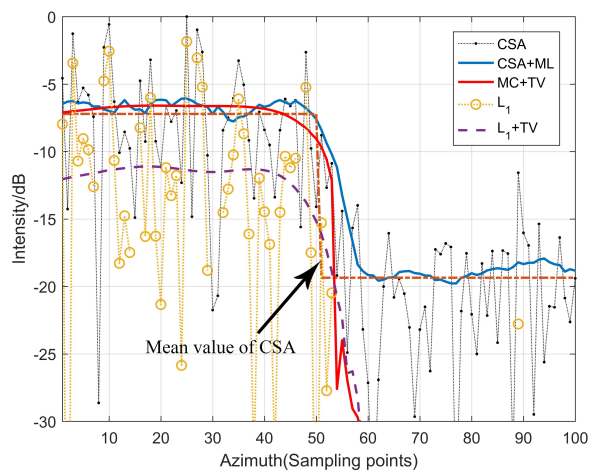

(a)

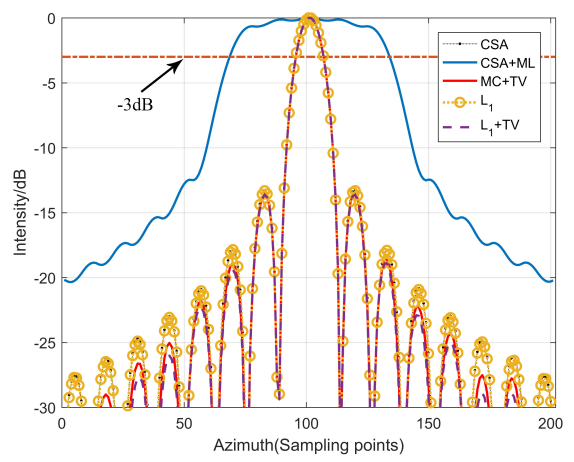

(b)

Figure 7. The slice along the azimuth direction of data 2. (a) The edge of the rainforest. (b) The simulated point target.

\section{Discussion}

In this section, we will explore the effectiveness of nonconvex and TV regularization under the condition of downsampling. We first perform $80 \%$ random downsampling in the azimuth direction for the fully sampled GF-3 echo data 1, and the scene corresponding to data 1 is shown in Figure 4a. Figure 8 shows the reconstructed images from downsampled 
echo data by CSA, CSA + multilook processing using a 5 by 5 window, $L_{1}$ regularization, $L_{1}$ and TV regularization and the proposed nonconvex and TV regularization, respectively. All the images in Figure 8 are plotted with the same color map. It can be seen that there are obvious ambiguities and energy dispersion along the azimuth direction due to the data downsampling, especially for targets with strong scattering intensity such as the target surrounded by a blue dotted rectangle in Figure 8. However, the latter three sparse SAR imaging methods reconstruct the considered scene successfully and simultaneously obtain an image with lower noise and clutter. Besides, it is obvious that nonconvex and TV regularization and $L_{1}$ and TV regularization can also maintain the uniformity and continuity of the backscattering coefficient in farmland regions.

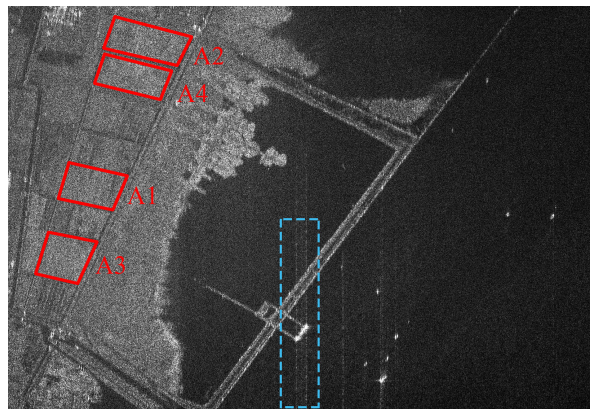

(a)

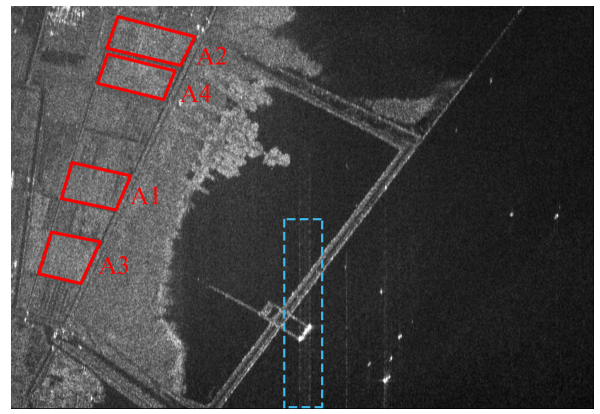

(b)

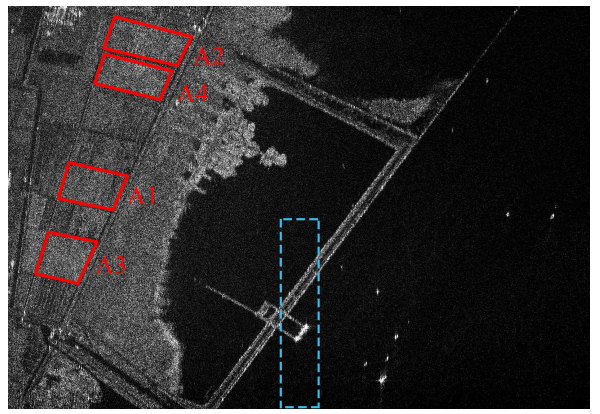

(c)

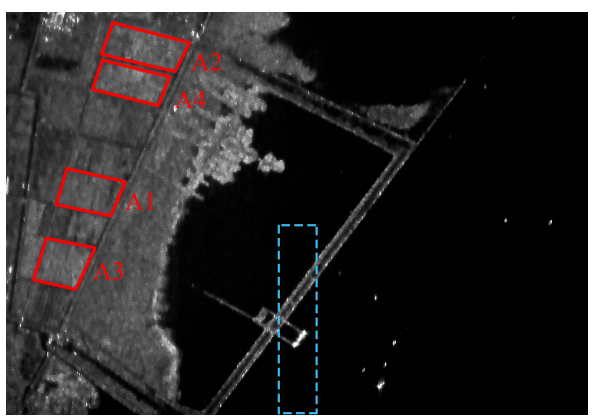

(d)

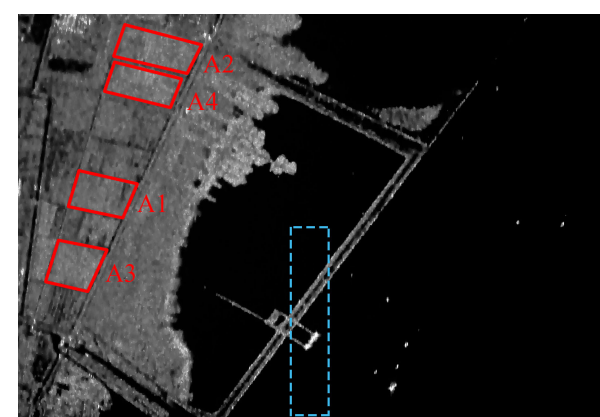

(e)

Figure 8. Experimental results for data 1 under downsampling condition. (a) Chirp scaling. (b) CSA + Multilook processing using a 5 by 5 window. (c) $L_{1}$ regularization. (d) $L_{1}$ and TV regularization. (e) Nonconvex and TV regularization. The vertical direction of the image is the azimuth direction, and the horizontal direction is the range direction. SAR data 1 occupies 1404 (azimuth) $\times 3941$ (range) sampling points, and the size of the corresponding scene is $8673 \mathrm{~m}$ (azimuth) $\times$ $8867 \mathrm{~m}$ (range). All the images are plotted with the same color map.

To quantitatively analyze the reconstruction accuracy and radiometric resolution, we select four regions as experimental distributed targets, which are the same as those in Experiment 1, and calculate the mean value, relative bias, variance, ENL, and radiometric resolution of the four targets. Statistical results in Table 3 show that under the condition of downsampling, nonconvex and TV regularization can also reconstruct targets at almost the same amplitude as the matched filtering method, as well as improve the radiometric resolution. 
Table 3. The reconstruction accuracy and radiometric resolution of four distributed targets in downsampling experiments based on data 1 for the five methods.

\begin{tabular}{ccccccc}
\hline Method & $\boldsymbol{\mu}$ & $\boldsymbol{R B}_{\boldsymbol{d}}$ & $\boldsymbol{\sigma}^{\mathbf{2}}$ & ENL & $\boldsymbol{\gamma}(\mathbf{d B})$ & Target \\
\hline CSA & 9.8115 & $/$ & 27.237 & 0.9657 & 3.0484 & \\
CSA + ML & 9.8021 & $/$ & 1.8626 & 14.094 & 1.0256 & $\mathrm{~A} 1$ \\
$L_{1}$ & 6.9433 & 0.2923 & 37.382 & 0.3524 & 4.2889 & \\
$L_{1}$ \& TV & 6.0151 & 0.3869 & 1.1548 & 8.5602 & 1.2768 & \\
MC \& TV & $\mathbf{9 . 1 4 1 8}$ & $\mathbf{0 . 0 6 8 3}$ & $\mathbf{1 . 4 1 6 4}$ & $\mathbf{1 6 . 1 2 0}$ & $\mathbf{0 . 9 6 5 9}$ & \\
\hline CSA & 10.073 & $/$ & 29.682 & 0.9339 & 3.0852 & \\
CSA + ML & 10.068 & $/$ & 2.5807 & 10.730 & 1.1570 & A2 \\
$L_{1}$ & 7.3991 & 0.2655 & 42.103 & 0.3553 & 4.2777 & \\
$L_{1} \&$ TV & 6.5702 & 0.3477 & 1.8127 & 6.5064 & 1.4365 & \\
MC \& TV & $\mathbf{9 . 7 9 3 9}$ & $\mathbf{0 . 0 2 7 7}$ & $\mathbf{1 . 7 3 8 5}$ & $\mathbf{1 5 . 0 7 5}$ & $\mathbf{0 . 9 9 5 3}$ & \\
\hline CSA & 10.340 & $/$ & 29.943 & 0.9755 & 3.0373 & \\
CSA + ML & 10.334 & $/$ & 2.1585 & 13.516 & 1.0449 & A3 \\
$L_{1}$ & 7.8030 & 0.2454 & 43.340 & 0.3838 & 4.1732 & \\
$L_{1} \&$ TV & 6.7917 & 0.3432 & 1.5232 & 8.2740 & 1.2958 & \\
MC \& TV & $\mathbf{9 . 9 8 4 5}$ & $\mathbf{0 . 0 3 4 4}$ & $\mathbf{1 . 3 4 6 5}$ & $\mathbf{2 0 . 2 2 7}$ & $\mathbf{0 . 8 7 1 9}$ & \\
\hline CSA & 10.726 & $/$ & 32.826 & 0.9576 & 3.0576 & \\
CSA + ML & 10.725 & $/$ & 2.6600 & 11.815 & 1.1090 & A4 \\
$L_{1}$ & 8.1305 & 0.2420 & 48.634 & 0.3714 & 4.2176 & \\
$L_{1} \&$ TV & 7.2285 & 0.3261 & 1.8624 & 7.6652 & 1.3392 & \\
MC \& TV & $\mathbf{1 0 . 5 5 7}$ & $\mathbf{0 . 0 1 5 8}$ & $\mathbf{1 . 7 4 3 6}$ & $\mathbf{1 7 . 4 6 3}$ & $\mathbf{0 . 9 3 1 8}$ & \\
\hline
\end{tabular}

Finally, we design a set of simulation experiments to verify the reconstruction performance of the proposed method under different downsampling ratios. The size of the simulated scene is $1024 \times 1024$ pixels. We place a distributed target that occupies $101 \times 101$ pixels at the center of the scene. According to Oliver and Quegan [24], we set an equivalent phase center in each pixel cell of the target, whose amplitudes are i.i.d. Rayleigh distributed (the mean value: $\mu=\sqrt{\pi \sigma_{0}} / 2$, the variance: $\sigma^{2}=\sqrt{(1-\pi / 4) \sigma_{0}}, \sigma_{0}$ : the backscattering coefficient) and phases are i.i.d. uniformly distributed $U[-\pi,+\pi)$. In this experiment, the SNR is set to $20 \mathrm{~dB}$, the range of the azimuth downsampling ratio is from $80 \%$ to $30 \%$, and the interval is $10 \%$. Under each downsampling ratio condition, we use the chirp scaling algorithm and the proposed method to reconstruct the target scene, and the imaging results are shown in Figure 9. Based on the above experimental results, we calculate the relative bias of the reconstruction results of the proposed method, as shown in Table 4, to quantitatively analyze the influence of the downsampling ratio on imaging performance. According to the calculation results in Table 4 and the imaging results in Figure 9, we can find the following: first, the amount of missing data will cause degradation of image quality, as the amount of missing data increases (the downsampling ratio decreases), the reconstruction performance becomes worse and the reconstruction bias of the proposed method gradually increases; second, as the amount of missing data increases, the probability of successful reconstruction gradually decreases, and under the conditions of this experiment, the proposed method will not be able to reconstruct the target scene successfully when the downsampling ratio is less than $40 \%$.

Table 4. The relative bias of the simulated distributed target under different downsampling ratios for the proposed method.

\begin{tabular}{ccccccc}
\hline \multirow{2}{*}{ MC \& TV } & \multicolumn{7}{c}{ Downsampling Ratio } \\
\cline { 2 - 7 } & $\mathbf{8 0} \%$ & $\mathbf{7 0} \%$ & $\mathbf{6 0} \%$ & $\mathbf{5 0} \%$ & $\mathbf{4 0} \%$ & $\mathbf{3 0} \%$ \\
\hline$R B_{d}$ & 0.0241 & 0.0276 & 0.0357 & 0.0704 & 0.1195 & 0.2402 \\
\hline
\end{tabular}




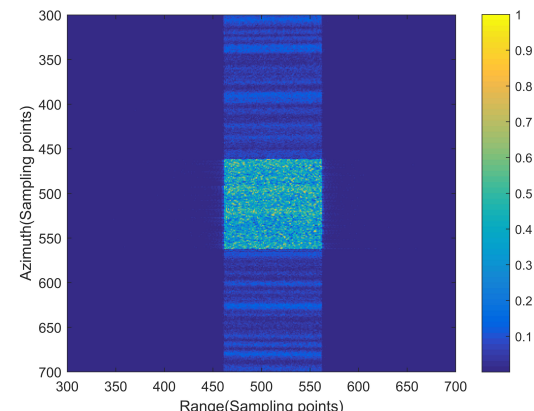

(a)

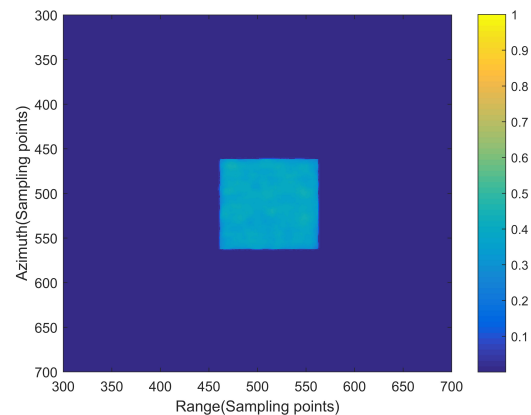

(d)

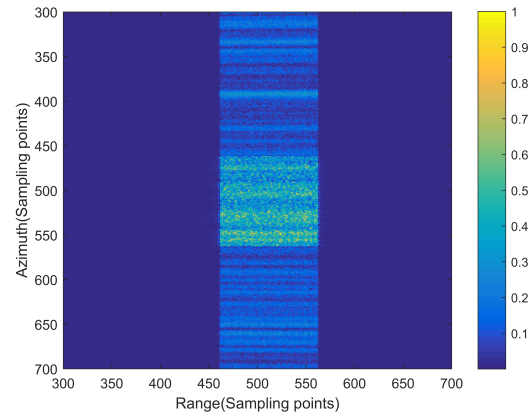

(g)

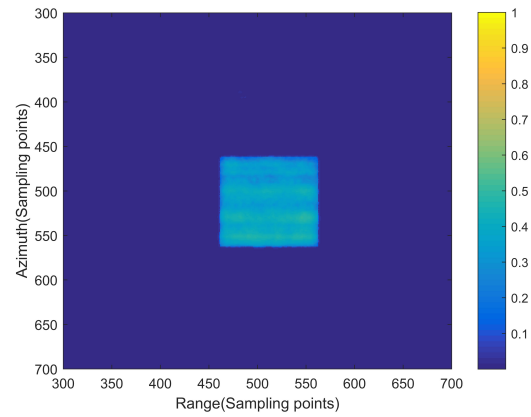

(j)

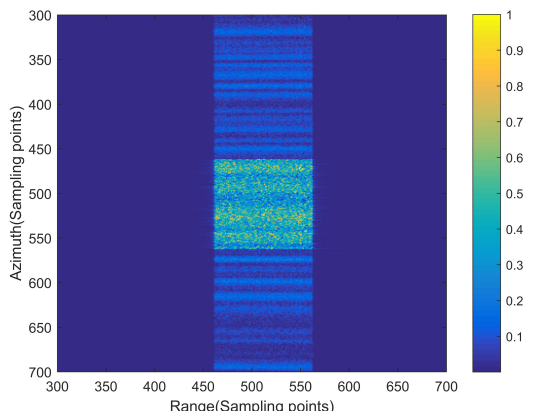

(b)

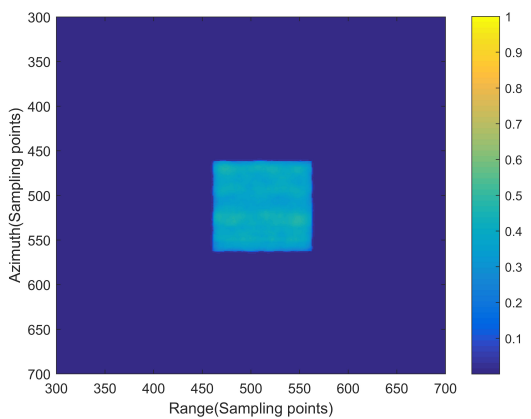

(e)

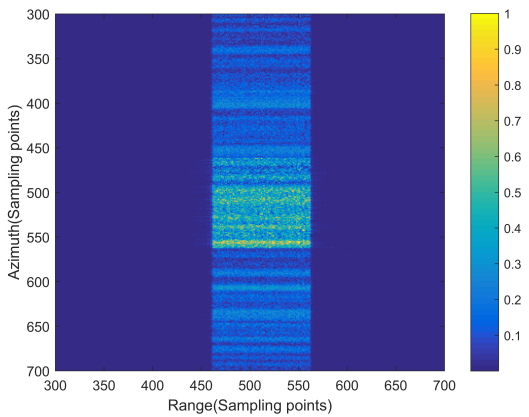

(h)

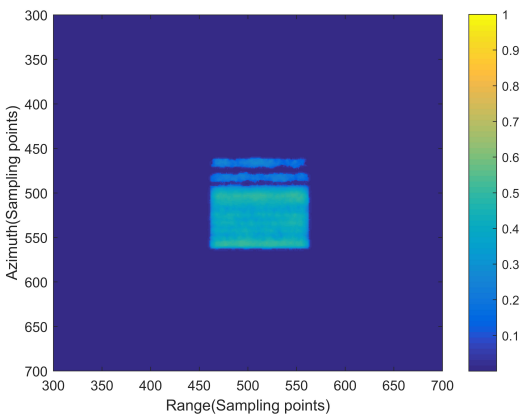

(k)

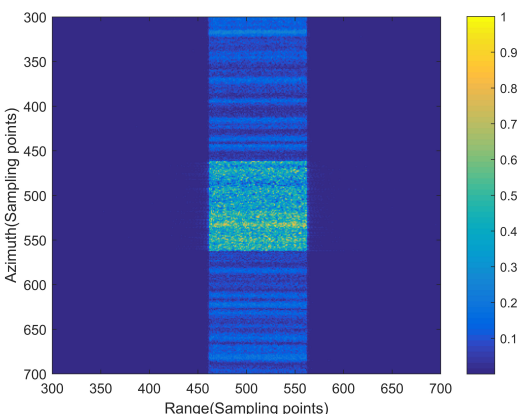

(c)

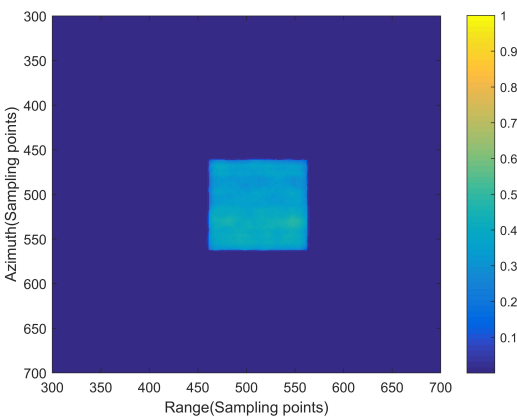

(f)

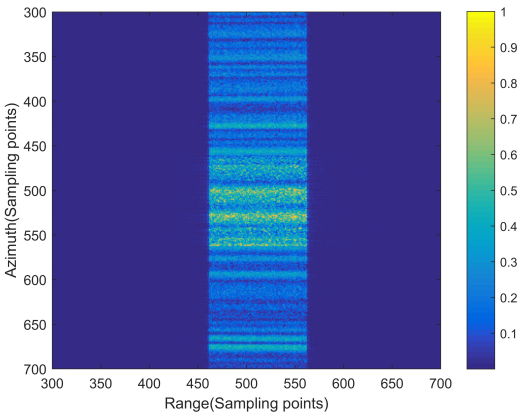

(i)

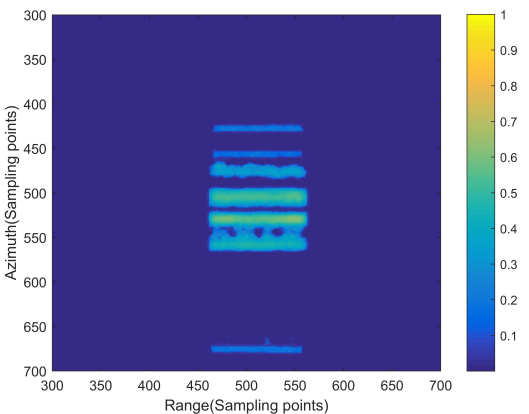

(1)

Figure 9. Simulation results under different downsampling conditions. (a-c,g-i): the reconstruction results of the chirp scaling algorithm. $(\mathbf{d}-\mathbf{f}, \mathbf{j}-\mathbf{l})$ : the reconstruction results of the proposed method. The top two rows: from left to right the ratios of azimuth downsampling are $80 \%, 70 \%$ and $60 \%$. The bottom two rows: from left to right the ratios of azimuth downsampling are $50 \%, 40 \%$ and $30 \%$. 


\section{Conclusions}

In this paper, we linearly combined the nonconvex penalty and the TV-norm penalty as a compound regularizer in the imaging model, called nonconvex and TV regularization, and proposed a novel algorithm combining the variable splitting scheme and the modified ADMM to solve it. To quantitatively evaluate the reconstructed SAR image quality based on nonconvex and TV regularization, we analyzed the radiometric property of sparsesignal-processing-based SAR imaging results and introduced three indexes suitable for sparse SAR imaging. Experimental results and image quality analysis based on GF-3 data verified the effectiveness of the proposed method in improving the reconstruction accuracy and the radiometric resolution without sacrificing the spatial resolution, which is beneficial to the quantitative use of SAR data, such as RCS estimation, target classification, and image segmentation. Based on the content of this paper, our next research work includes two aspects. The first is to optimize the TV-norm constraints (such as weighting processing) to improve the reconstruction performance of the method proposed in this paper. The second is to extend the method to the data processing of 3-D SAR imaging such as downward-looking array 3-D SAR and tomoSAR to improve the performance of 3-D reconstruction.

Author Contributions: Conceptualization, Z.X., B.Z. and Y.W.; methodology, Z.X.; validation, Z.X., G.Z. and L.Z.; formal analysis, Z.X.; investigation, Z.X.; resources, L.Z.; data curation, Z.X.; writingoriginal draft preparation, Z.X.; writing—review and editing, Z.X.; visualization, Z.X.; supervision, $Z$ Z.X. All authors have read and agreed to the published version of the manuscript.

Funding: This research was funded by the National Natural Science Foundation of China Grant No. 61991421.

Informed Consent Statement: Informed consent was obtained from all subjects involved in the study.

Acknowledgments: The authors would like to thank the China Center for Resources Satellite Data and Application for providing the GF-3 data.

Conflicts of Interest: The authors declare no conflict of interest.

\section{References}

1. Ender, J.H. On compressive sensing applied to radar. Signal Process. 2010, 90, 1402-1414. [CrossRef]

2. Zhang, B.; Hong, W.; Wu, Y. Sparse microwave imaging: Principles and applications. Sci. China Inf. Sci. 2012, 55, 1722-1754. [CrossRef]

3. Cetin, M.; Stojanovic, I.; Onhon, O.; Varshney, K.; Samadi, S.; Karl, W.C.; Willsky, A.S. Sparsity-driven synthetic aperture radar imaging: Reconstruction, autofocusing, moving targets, and compressed sensing. IEEE Signal Process. Mag. 2014, 31, 27-40. [CrossRef]

4. Ao, D.; Wang, R.; Hu, C.; Li, Y. A Sparse SAR Imaging Method Based on Multiple Measurement Vectors Model. Remote Sens. 2017, 9, 297. [CrossRef]

5. Bi, H.; Zhang, B.; Zhu, X.X.; Jiang, C.; Hong, W. Extended Chirp Scaling-Baseband Azimuth Scaling-Based Azimuth-Range Decouple $L_{1}$ Regularization for TOPS SAR Imaging via CAMP. IEEE Trans. Geosci. Remote Sens. 2017, 55, 3748-3763. [CrossRef]

6. Baraniuk, R.; Steeghs, P. Compressive radar imaging. In Proceedings of the 2007 IEEE Radar Conference, Waltham, MA, USA, 17-20 April 2007; pp. 128-133.

7. Alonso, M.T.; López-Dekker, P.; Mallorquí, J.J. A novel strategy for radar imaging based on compressive sensing. IEEE Trans. Geosci. Remote Sens. 2010, 48, 4285-4295. [CrossRef]

8. Patel, V.M.; Easley, G.R.; Healy, D.M., Jr.; Chellappa, R. Compressed synthetic aperture radar. IEEE J. Sel. Top. Signal Process. 2010, 4, 244-254. [CrossRef]

9. Osher, S.; Ruan, F.; Xiong, J.; Yao, Y.; Yin, W. Sparse recovery via differential inclusions. Appl. Comput. Harmon. Anal. 2016, 41, 436-469. [CrossRef]

10. Candes, E.; Tao, T. The Dantzig selector: Statistical estimation when p is much larger than n. Ann. Stat. 2007, 35, $2313-2351$.

11. Güven, H.E.; Güngör, A.; Cetin, M. An augmented lagrangian method for complex-valued compressed sar imaging. IEEE Trans. Comput. Imaging 2016, 2, 235-250. [CrossRef]

12. Selesnick, I. Sparse regularization via convex analysis. IEEE Trans. Signal Process. 2017, 65, 4481-4494. [CrossRef]

13. Wei, Z.; Zhang, B.; Wu, Y. A SAR imaging method based on generalized minimax-concave penalty. Sci. China Inf. Sci. 2019, 62, 1-3. [CrossRef] 
14. Gong, P.; Zhang, C.; Lu, Z.; Huang, J.; Ye, J. A general iterative shrinkage and thresholding algorithm for non-convex regularized optimization problems. In Proceedings of the International Conference on Machine Learning, Atlanta, GA, USA, 16-21 June 2013; pp. 37-45.

15. Ugur, S.; Arıkan, O. SAR image reconstruction and autofocus by compressed sensing. Digit. Signal Process. 2012, $22,923-932$. [CrossRef]

16. Zhao, Y.; Liu, J.G.; Zhang, B.; Hong, W.; Wu, Y.R. Adaptive total variation regularization based SAR image despeckling and despeckling evaluation index. IEEE Trans. Geosci. Remote Sens. 2014, 53, 2765-2774. [CrossRef]

17. Kang, M.S.; Kim, K.T. Compressive sensing based SAR imaging and autofocus using improved Tikhonov regularization. IEEE Sens. J. 2019, 19, 5529-5540. [CrossRef]

18. Xu, Z.; Liu, M.; Zhou, G.; Wei, Z.; Zhang, B.; Wu, Y. An Accurate Sparse SAR Imaging Method for Enhancing Region-Based Features Via Nonconvex and TV Regularization. IEEE J. Sel. Top. Appl. Earth Obs. Remote Sens. 2021, 14, 350-363. [CrossRef]

19. Zhang, Y.; Miao, W.; Lin, Z.; Gao, H.; Shi, S. Millimeter-Wave InSAR Image Reconstruction Approach by Total Variation Regularized Matrix Completion. Remote Sens. 2018, 10, 1053. [CrossRef]

20. Afonso, M.V.; Bioucas-Dias, J.M.; Figueiredo, M.A.T. Fast Image Recovery Using Variable Splitting and Constrained Optimization. IEEE Trans. Image Process. 2010, 19, 2345-2356. [CrossRef] [PubMed]

21. Afonso, M.V.; Bioucas-Dias, J.M.; Figueiredo, M.A.T. An Augmented Lagrangian Approach to the Constrained Optimization Formulation of Imaging Inverse Problems. IEEE Trans. Image Process. 2011, 20, 681-695. [CrossRef] [PubMed]

22. Chen, Q.; Li, Z.; Zhang, P.; Tao, H.; Zeng, J. A preliminary evaluation of the GaoFen-3 SAR radiation characteristics in land surface and compared with Radarsat-2 and Sentinel-1A. IEEE Geosci. Remote Sens. Lett. 2018, 15, 1040-1044. [CrossRef]

23. Mittermayer, J.; Younis, M.; Metzig, R.; Wollstadt, S.; Martínez, J.M.; Meta, A. TerraSAR-X system performance characterization and verification. IEEE Trans. Geosci. Remote Sens. 2009, 48, 660-676. [CrossRef]

24. Oliver, C.; Quegan, S. Understanding Synthetic Aperture Radar Images; SciTech Publishing: Raleigh, NC, USA, 2004.

25. Cozzolino, D.; Verdoliva, L.; Scarpa, G.; Poggi, G. Nonlocal CNN SAR Image Despeckling. Remote Sens. 2020, 12, 1006. [CrossRef]

26. Chambolle, A. An algorithm for total variation minimization and applications. J. Math. Imaging Vis. 2004, 20, 89-97.

27. Fang, J.; Xu, Z.; Zhang, B.; Hong, W.; Wu, Y. Fast compressed sensing SAR imaging based on approximated observation. IEEE J. Sel. Top. Appl. Earth Obs. Remote Sens. 2013, 7, 352-363. [CrossRef]

28. Potter, L.C.; Ertin, E.; Parker, J.T.; Cetin, M. Sparsity and compressed sensing in radar imaging. Proc. IEEE 2010, 98, 1006-1020. [CrossRef]

29. Lee, J.S.; Jurkevich, L.; Dewaele, P.; Wambacq, P.; Oosterlinck, A. Speckle filtering of synthetic aperture radar images: A review. Remote Sens. Rev. 1994, 8, 313-340. [CrossRef]

30. Raney, R.K.; Runge, H.; Bamler, R.; Cumming, I.G.; Wong, F.H. Precision SAR processing using chirp scaling. IEEE Trans. Geosci. Remote Sens. 1994, 32, 786-799. [CrossRef] 